\title{
Estimation of the Inverse Weibull Distribution Parameters under Type-I Hybrid Censoring
}

\author{
Mohammad Kazemi \\ University of Guilan
}

\author{
Mina Azizpoor \\ University of Mazandaran
}

\begin{abstract}
The hybrid censoring is a mixture of type-I and type-II censoring schemes. This paper presents the statistical inferences of the inverse Weibull distribution parameters when the data are type-I hybrid censored. First, we consider the maximum likelihood estimates of the unknown parameters. It is observed that the maximum likelihood estimates can not be obtained in closed form. We further obtain the Bayes estimates and the corresponding highest posterior density credible intervals of the unknown parameters under the assumption of independent gamma priors using the importance sampling procedure. We also compute the approximate Bayes estimates using Lindley's approximation technique. The performance of the Bayes estimates have been compared with maximum likelihood estimates through the Monte Carlo Markov chain techniques. Finally, a real data set have been analysed for illustration purpose.
\end{abstract}

Keywords: Bayes estimators, hybrid censoring, importance sampling, maximum likelihood estimators.

\section{Introduction}

Type-I and type-II are the two most popular censoring schemes which are in use for any life testing experiment. Two mixtures of type-I and type-II censoring schemes are known as hybrid censoring schemes. If the experiment terminates as soon as either the $R$-th failure or the pre-specified censoring time $T$ occurs, type-I hybrid censoring scheme has been performed. In type-II hybrid censoring scheme, the experiment terminates when the latter of the $R$-th failure and the censoring time $T$ occurs. Denote the $i$-th order statistic from a random sample of size $n$ by $X_{i: n}$. Thus, in type-I hybrid censoring scheme, one observes $X_{1: n}, \cdots, X_{r: n}$ when $X_{r: n} \leq \min \left\{X_{R: n}, T\right\}$ and $X_{r+1: n}>\min \left\{X_{R: n}, T\right\}$. Under this scheme, the experiment may be terminated too early resulting in very few failures. Under type-II hybrid censoring scheme, the experiment terminates when $X_{1: n}, \cdots, X_{r: n}$ are observed for which $X_{r: n} \leq \max \left\{X_{R: n}, T\right\}$ and $X_{r+1: n}>\max \left\{X_{R: n}, T\right\}$. In both hybrid censoring schemes, the failure number $R$ and censoring time $T$ are pre-fixed.

Epstein (1954) first introduced the hybrid censoring scheme and analyzed the data under the assumption of exponential lifetime distribution of the experimental units. An extensive literature exists for hybrid censoring under classical and Bayesian framework and the overview 
presented below describe some of the work done on this topic. Gupta and Kundu (1988) obtained confidence and credible intervals for an one-parameter exponential distribution. Kundu (2007) obtained the MLE's, the approximate MLE's and Bayes estimates of shape and scale parameters of a Weibull distribution. Kundu and Pradhan (2009) analyzed a generalized exponential distribution in presence of hybrid censoring. Balakrishnan and Shafay (2012) developed a general method for obtaining Bayes prediction intervals of future observable based on an observed type-I hybrid censored data. Rastogi and Tripathi (2013) derived maximum likelihood and Bayes estimates of the unknown model parameters of a Burr XII distribution. Singh and Tripathi (2015) studied a two-parameter lognormal distribution using hybrid censored samples and derived various point and interval estimates of unknown lognormal parameters from classical and Bayesian viewpoint. Tripathi and Rastogi (2016) considered point and interval estimation of the unknown parameters of a generalized inverted exponential distribution and obtained various classical and Bayes estimates based on hybrid censored samples. Hyun, Lee, and Yearout (2016) analyzed a two-parameter log-logistic distribution based on type I and type II hybrid censored data.

In this paper, we provide point and interval estimates for the unknown parameters of an inverse Weibull (IW) distribution based on type-I hybrid censored samples. The probability density function (PDF) of an IW distribution is

$$
f_{X}(x ; \alpha, \lambda)=\frac{\alpha}{\theta^{\alpha}} x^{-(\alpha+1)} e^{-(\theta x)^{-\alpha}}, \quad x>0, \alpha>0, \theta>0
$$

and the corresponding cumulative distribution function $(\mathrm{CDF})$ is given by

$$
F_{X}(x ; \alpha, \lambda)=e^{-(\theta x)^{-\alpha}}, \quad x>0, \alpha>0, \theta>0,
$$

where $\alpha$ and $\theta$ are the shape and scale parameters respectively. As in the Weibull distribution, the shape parameter $\alpha$ governs the shape of the PDF, the hazard function and the general properties of the IW distribution. When $\alpha=1$ and $\alpha=2$, the IW distribution reduce to the inverse exponential and inverse Rayleigh distributions respectively.

Extensive work has been done on the IW distribution. Kundu and Howlader (2010) considered the Bayesian inference and prediction problems of the IW distribution based on type-II censored data. Singh, Singh, and Sharma (2013) proposed a Bayesian procedure for the estimation of the parameters of IW distribution under type-II hybrid censoring scheme. Ateya (2017) and Ateya (2020) considered estimation of the unknown parameters of the IW distribution based on Balakrishnan's unified hybrid censoring and generalized type-II progressive hybrid censoring schemes, respectively. We consider the inference for IW distribution under type-I hybrid censoring scheme.

The rest of the paper is organized as follows. In Section 2, we discuss the maximum likelihood estimation of the scale and shape parameters of the IW distribution. The asymptotic confidence bounds are provided in section 3. Bayesian analyses are presented in Section 4. In Section 5, we conduct a simulation study to compare the performance of proposed methods and then analyzed a real data set for illustrative purpose in Section 6. Finally we conclude the paper in section 7 .

\section{Maximum likelihood estimation}

In this section we provide the maximum likelihood estimators (MLEs) of the unknown parameters. We re-parametrize the model as follows $\lambda=\frac{1}{\theta^{\alpha}}$. Suppose $n$ identical units are put on life test. Then under type-I hybrid censoring scheme, we observe only the first $r$ failure times, say $t_{1}, t_{2}, \ldots, t_{r}$. Under the assumptions that the lifetime distribution of the items are independent and identically distributed (i.i.d.) IW random variable, the likelihood function 
for the type-I hybrid censored data without the multiplicative constant can be written as

$$
L(\alpha, \lambda \mid \text { data })=\alpha^{r} \lambda^{r} e^{-\lambda \sum_{i=1}^{r} x_{i}^{\alpha}} \prod_{i=1}^{r} x_{i}^{\alpha+1}\left(1-e^{-\lambda u^{-\alpha}}\right)^{n-r}, \quad \alpha>0, \lambda>0,
$$

where $x_{i}=\frac{1}{t_{(i)}}, u=\min \left(t_{(R)}, T\right)$ and $r$ denotes the number of units that would fail before the time $u$. Taking the logarithm of (3), we obtain

$$
l(\alpha, \lambda \mid \text { data })=r \ln (\alpha \lambda)-\lambda \sum_{i=1}^{r} x_{i}^{\alpha}+(\alpha+1) \sum_{i=1}^{r} \ln x_{i}+(n-r) \ln \left(1-e^{-\lambda u^{-\alpha}}\right) .
$$

Taking derivatives with respect to $\alpha$ and $\lambda$ of (4), and equality to zero, we obtain

$$
\begin{aligned}
& \frac{\partial \ln L}{\partial \alpha}=\frac{r}{\alpha}-\lambda \sum_{i=1}^{r} x_{i}^{\alpha} \ln x_{i}+\sum_{i=1}^{r} \ln x_{i}+(n-r) \frac{\lambda u^{-\alpha} \ln u e^{-\lambda u^{-\alpha}}}{1-e^{-\lambda u^{-\alpha}}}=0, \\
& \frac{\partial \ln L}{\partial \lambda}=\frac{r}{\lambda}-\sum_{i=1}^{r} x_{i}^{\alpha}+(n-r) \frac{u^{-\alpha} e^{-\lambda u^{-\alpha}}}{1-e^{-\lambda u^{-\alpha}}}=0 .
\end{aligned}
$$

It is clear that the normal equations do not have explicit solutions. We need some numerical techniques to solve the simultaneous equations.

\section{Asymptotic confidence bounds}

Since the MLEs of the unknown parameters $\alpha, \lambda$ can not be obtained in closed forms, it is not easy to derive the exact distributions of the MLEs. Therefore, the exact confidence intervals for the unknown parameters is difficult to obtain. In this section, we compute the observed Fisher information based on the likelihood equations. These will enable us to develop pivotal quantities based on the limiting normal distribution and then construct asymptotic confidence intervals.

From the log-likelihood function in (4) we obtain the observed Fisher information as

$$
\begin{aligned}
\frac{\partial^{2} l(\alpha, \lambda)}{\partial \alpha^{2}} & =-\frac{r}{\alpha^{2}}-\lambda \sum_{i=1}^{r} x_{i}^{\alpha}\left(\ln x_{i}\right)^{2}+\frac{(n-r) \lambda u^{-\alpha}(\ln u)^{2} e^{-\lambda u^{-\alpha}}\left(1-\lambda u^{-\alpha}\right)}{1-e^{-\lambda u^{-\alpha}}} \\
& -\frac{(n-r) \lambda^{2} u^{-2 \alpha} \ln u^{2} e^{-2 \lambda u^{-\alpha}}}{\left(1-e^{-\lambda u^{-\alpha}}\right)^{2}}, \\
\frac{\partial^{2} l(\alpha, \lambda)}{\partial \alpha \partial \lambda} & =-\sum_{i=1}^{r} x_{i}^{\alpha} \ln x_{i}-\frac{(n-r) u^{-\alpha} \ln (u) e^{-\lambda u^{-\alpha}}\left(1-\lambda u^{-\alpha}\right)}{1-e^{-\lambda u^{-\alpha}}} \\
& +\frac{(n-r) u^{-2 \alpha} \ln (u) \lambda e^{-2 \lambda u^{-\alpha}}}{\left(1-e^{-\lambda u^{-\alpha}}\right)^{2}}, \\
\frac{\partial^{2} l(\alpha, \lambda)}{\partial \lambda^{2}} & =-\frac{r}{\lambda^{2}}-\frac{(n-r) u^{-2 \alpha} e^{-\lambda u^{-\alpha}}}{1-e^{-\lambda u^{-\alpha}}}-\frac{(n-r) u^{-2 \alpha} e^{-2 \lambda u^{-\alpha}}}{\left(1-e^{-\lambda u^{-\alpha}}\right)^{2}} .
\end{aligned}
$$

The observed Fisher information matrix can be inverted to obtain the asymptotic variancecovariance matrix of the MLEs as

$$
\mathbf{I}^{-1}=\left\{-\left(\begin{array}{cc}
\left.\frac{\partial^{2} l(\alpha, \lambda)}{\partial \alpha^{2}}\right|_{\hat{\alpha}, \hat{\lambda}} & \left.\frac{\partial^{2} l(\alpha, \lambda)}{\partial \alpha \partial \lambda}\right|_{\hat{\alpha}, \hat{\lambda}} \\
\left.\frac{\partial^{2} l(\alpha, \lambda)}{\partial \alpha \partial \lambda}\right|_{\hat{\alpha}, \hat{\lambda}} & \left.\frac{\partial^{2} l(\alpha, \lambda)}{\partial \lambda^{2}}\right|_{\hat{\alpha}, \hat{\lambda}}
\end{array}\right)\right\}^{-1}=\left(\begin{array}{cc}
V^{11} & V^{12} \\
V^{12} & V^{22}
\end{array}\right) .
$$


It is well known that MLEs are asymptotically normally distributed and using this property of MLEs, we can construct the approximate confidence intervals for $\alpha$ and $\lambda$. Since the $\hat{\alpha}$ and $\hat{\lambda}$ is asymptotically normally distributed, we have the asymptotic distribution of

$$
P_{1}=\frac{\hat{\alpha}-\alpha}{\sqrt{V^{11}}}, \quad P_{2}=\frac{\hat{\lambda}-\lambda}{\sqrt{V^{22}}},
$$

to be standard normal. Using the pivotal quantities $P_{1}$ and $P_{2}, 100(1-\gamma) \%$ asymptotic confidence intervals for $\alpha$ and $\lambda$ based on the MLEs are

$$
\left(\widehat{\alpha}-z_{\frac{\gamma}{2}} \sqrt{V^{11}}, \widehat{\alpha}+z_{\frac{\gamma}{2}} \sqrt{V^{11}}\right),
$$

and

$$
\left(\widehat{\lambda}-z_{\frac{\gamma}{2}} \sqrt{V^{22}}, \widehat{\lambda}+z_{\frac{\gamma}{2}} \sqrt{V^{22}}\right),
$$

respectively, where $z_{\frac{\gamma}{2}}$ is the $\left(\frac{\gamma}{2}\right)^{t h}$ upper percentile of standard normal distribution.

\section{Bayesian analysis}

In this section we compute the Bayes estimates and the associated HPD credible intervals of the shape and scale parameters. We need to assume some prior distributions of the unknown parameters for the Bayesian inference. Unfortunately, when both the parameters are unknown, there does not exist any natural conjugate priors. In this paper similarly as in Kundu and Howlader (2010), it is assumed that $\alpha$ and $\lambda$ have the following independent gamma priors;

$$
\begin{aligned}
& \pi(\alpha \mid a, b) \propto \alpha^{a-1} e^{-b \alpha}, \quad \alpha>0, \\
& \pi(\lambda \mid c, d) \propto \lambda^{c-1} e^{-d \lambda}, \quad \lambda>0 .
\end{aligned}
$$

Here all the hyper parameters $a, b, c, d$ are assumed to be known and positive. Based on the above priors, the joint density function of the data, $\alpha$ and $\lambda$ is

$$
L(\text { data }, \alpha, \lambda)=\alpha^{r+a-1} \lambda^{r+c-1} e^{-b \alpha-\lambda\left\{d+\sum_{i=1}^{r} x_{i}^{\alpha}\right\}} \prod_{i=1}^{r} x_{i}^{\alpha+1}\left(1-e^{-\lambda u^{-\alpha}}\right)^{n-r} .
$$

Based on $L($ data, $\alpha, \lambda)$, we obtain the joint posterior density function of $\alpha$ and $\lambda$ given the data as

$$
\pi(\alpha, \lambda \mid \text { data })=\frac{L(\text { data }, \alpha, \lambda)}{\int_{0}^{\infty} \int_{0}^{\infty} L(\text { data }, \alpha, \lambda) d \alpha d \lambda} .
$$

Therefore, the posterior density function of $\alpha$ and $\lambda$ given the data can be written as

$$
\pi(\alpha, \lambda \mid \text { data }) \propto g_{1}(\lambda \mid \alpha, \text { data }) g_{2}(\alpha \mid \text { data }) h(\alpha, \lambda \mid \text { data }),
$$

here $g_{1}(\lambda \mid \alpha$, data $)$ is a gamma density function with the shape and scale parameters as $(r+c)$ and $\left(d+\sum_{i=1}^{r} x_{i}^{\alpha}\right)$, respectively. Also $g_{2}(\alpha \mid d a t a)$ is a proper density function given by

$$
g_{2}(\alpha \mid d a t a) \propto \frac{1}{\left(d+\sum_{i=1}^{r} x_{i}^{\alpha}\right)^{r+c}} \alpha^{a+r-1} e^{-b \alpha} \prod_{i=1}^{r} x_{i}^{\alpha+1} .
$$

Moreover

$$
h(\alpha, \lambda \mid \text { data })=\left(1-e^{-\lambda u^{-\alpha}}\right)^{n-r} .
$$

Therefore, the Bayes estimate of any function of $\alpha$ and $\lambda$, say $g(\alpha, \lambda)$ under the squared error loss function is

$$
\hat{g}_{B}(\alpha, \lambda)=\frac{\int_{0}^{\infty} \int_{0}^{\infty} g(\alpha, \lambda) g_{1}(\lambda \mid \alpha, \text { data }) g_{2}(\alpha \mid \text { data }) h(\alpha, \lambda \mid \text { data }) d \alpha d \lambda}{\int_{0}^{\infty} \int_{0}^{\infty} g_{1}(\lambda \mid \alpha, \text { data }) g_{2}(\alpha \mid \text { data }) h(\alpha, \lambda \mid \text { data }) d \alpha d \lambda} .
$$


Unfortunately, (12) can not be computed analytically for general $g(\alpha, \lambda)$. We apply two different approximation methods to evaluate the Bayes estimators of $\alpha$ and $\lambda$. The first approximation technique due to Lindley (1980) and the second is an importance sampling procedure as suggested by Chen and Shao (1999). The details are explained below.

\subsection{Lindley's approximation}

It is known that the (12) can not be computed explicitly. Because of that Lindley (1980) proposed an approximation to compute the ratio of two integrals such as (12). This has been used by several authors to obtain the approximate Bayes estimators. This approximation technique uses Taylor's series expansion of the integral expression around maximum likelihood estimator.

Based on Lindley's approximation, the approximate Bayes estimates of $\alpha$ and $\lambda$ under the squared error loss functions are respectively

$$
\begin{aligned}
\hat{\alpha}_{L} & =\hat{\alpha}+\frac{1}{2}\left[l_{30} \tau_{11}^{2}+l_{03} \tau_{21} \tau_{22}+3 l_{21} \tau_{11} \tau_{12}+l_{12}\left(\tau_{22} \tau_{11}+2 \tau_{21}^{2}\right)\right] \\
& +\left(\frac{a-1}{\hat{\alpha}}-b\right) \tau_{11}+\left(\frac{c-1}{\hat{\lambda}}-d\right) \tau_{12} \\
\hat{\lambda}_{L} & =\hat{\lambda}+\frac{1}{2}\left[l_{30} \tau_{12} \tau_{11}+l_{03} \tau_{22}^{2}+l_{21}\left(\tau_{11} \tau_{22}+2 \tau_{12}^{2}\right)+3 l_{12} \tau_{22} \tau_{21}\right] \\
& +\left(\frac{a-1}{\hat{\alpha}}-b\right) \tau_{21}+\left(\frac{c-1}{\hat{\lambda}}-d\right) \tau_{22},
\end{aligned}
$$

where $\hat{\alpha}$ and $\hat{\lambda}$ are MLEs of $\alpha$ and $\lambda$ respectively and a, b, c, d are the known hyper parameters. The explicit expressions of $\tau_{11}, \tau_{12}, \tau_{22}, l_{30}, \tau_{21}, l_{03}, l_{12}, l_{21}$ are provided in the Appendix A.

Although using Lindley's approximation we can obtain the Bayes estimates, but it is not possible to construct the HPD credible intervals using this method. Therefore, in the next subsection we propose the importance sampling procedure to draw samples from the posterior density function and in turn compute the Bayes estimates, and also construct HPD credible intervals.

\subsection{Importance sampling}

We use importance sampling to generate a sample from the posterior density function $\pi(\alpha, \lambda \mid$ data) and then to compute the Bayes estimates and HPD credible intervals. The following theorem can be useful for further development.

Theorem 1. The conditional density of $\alpha$, given data, say $g_{2}(\alpha \mid$ data $)$ is log-concave.

Proof. See Appendix B.

Because of log-concavity of $g_{2}(\alpha \mid$ data $)$, the idea of Devroye (1984) can be used to generate a sample from $g_{2}(\alpha \mid$ data $)$. Moreover, since $g_{1}(\lambda \mid \alpha$, data $)$ follows gamma, it is quite simple to generate from $g_{1}(\lambda \mid \alpha$,data). Now we would like to provide the importance sampling procedure to compute the Bayes estimates and also to construct the credible interval of $g(\alpha, \beta)=\theta$ (say). Similarly as in Raqab and Madi (2005) a simulation based consistent estimate of $E(g(\alpha, \beta))=E(\theta)$ can be obtained using Algorithm as given below.

\section{Algorithm.}

Step 1: Generate $\alpha$ from $g_{2}(. \mid$ data $)$ using the method developed by Devroye (1984). 
Step 2: Generate $\lambda$ from $g_{1}(. \mid \alpha$, data $)$.

Step 3: Repeat Step 1 and Step 2 and obtain $\left(\alpha_{1}, \lambda_{1}\right), \ldots,\left(\alpha_{M}, \lambda_{M}\right)$.

Step 4: An approximate Bayes estimate of $\theta$ under a squared error loss function can be obtained as

$$
\hat{g}_{B}(\alpha, \lambda)=\hat{\theta}=\frac{\frac{1}{M} \sum_{i=1}^{M} \theta_{i} h\left(\alpha_{i}, \lambda_{i} \mid \text { data }\right)}{\frac{1}{M} \sum_{i=1}^{M} h\left(\alpha_{i}, \lambda_{i} \mid \text { data }\right)} .
$$

Step 5: Obtain the posterior variance of $g(\alpha, \beta)=\theta$ as

$$
\hat{V}(g(\alpha, \lambda \mid \text { data }))=\frac{\frac{1}{M} \sum_{i=1}^{M}\left(\theta_{i}-\hat{\theta}\right)^{2} h\left(\alpha_{i}, \lambda_{i} \mid \text { data }\right)}{\frac{1}{M} \sum_{i=1}^{M} h\left(\alpha_{i}, \lambda_{i} \mid \text { data }\right)} .
$$

We now obtain the credible interval of $\theta$ using the idea of Chen and Shao (1999). Let us denote $\pi(\theta \mid$ data $)$ and $\Pi(\theta \mid$ data $)$ as the posterior density and posterior distribution functions of $\theta$, respectively. Also let $\theta^{(\beta)}$ be the $\beta$-th quantile of $\theta$, i.e.,

$$
\theta^{(\beta)}=\inf \{\theta ; \Pi(\theta \mid \text { data }) \geq \beta\}, \quad 0<\beta<1 .
$$

Observe that for a given $\theta^{*}, \Pi\left(\theta^{*} \mid\right.$ data $)=E\left\{I_{\theta \leq \theta^{*}} \mid\right.$ data $\}$, where $I_{\theta \leq \theta^{*}}$ is the indicator function. Therefore, a simulation consistent estimator of $\Pi\left(\theta^{*} \mid\right.$ data $)$ can be obtained as

$$
\hat{\Pi}\left(\theta^{*} \mid \text { data }\right)=\frac{\frac{1}{M} \sum_{i=1}^{M} I_{\theta_{i} \leq \theta^{*}} h\left(\alpha_{i}, \lambda_{i} \mid \text { data }\right)}{\frac{1}{M} \sum_{i=1}^{M} h\left(\alpha_{i}, \lambda_{i} \mid \text { data }\right)} .
$$

For $i=1, \ldots, M$, let $\left\{\theta_{(i)}\right\}$ be the ordered values of $\theta_{i}$, and

$$
w_{i}=\frac{h\left(\alpha_{(i)}, \lambda_{(i)} \mid \text { data }\right)}{\sum_{i=1}^{M} h\left(\alpha_{i}, \lambda_{i} \mid \text { data }\right)},
$$

be the associated weight, then we have

$$
\hat{\Pi}\left(\theta^{*} \mid \text { data }\right)=\left\{\begin{array}{lrl}
0 & \theta^{*} & <\theta_{(1)} \\
\sum_{j=1}^{i} w_{j} & \theta_{(i)} & \leq \theta^{*}<\theta_{(i+1)} \\
1 & \theta^{*} & \geq \theta_{(M)} .
\end{array}\right.
$$

Therefore, $\theta^{(\beta)}$ can be approximated by

$$
\hat{\theta}^{(\beta)}= \begin{cases}\theta_{(1)} & \beta=0 \\ \theta_{(i)} & \sum_{j=1}^{i-1} w_{j}<\beta \leq \sum_{j=1}^{i} w_{j} .\end{cases}
$$

To obtain a $100(1-\beta) \%$ HPD credible interval for $\theta$, consider intervals of the form

$$
R_{j}=\left(\hat{\theta}^{\left(\frac{j}{M}\right)}, \hat{\theta}^{\left(\frac{j+[(1-\beta) M]}{M}\right)}\right)
$$

for $j=1,2, \ldots, M-[(1-\beta) M]$, where $[a]$ denotes the largest integer less than or equal to $[a]$. Finally, among all $R_{j}$ choose that interval which has the smallest length.

\section{Simulation studies}

In this section we compare the performance of the different methods through a simulation study. We estimate the unknown parameters using the MLE, Bayes estimators obtained by Lindley's approximations, and also by the Bayes estimators obtained by using MCMC technique. The simulation study is carried out for different sample size and with different 
Table 1: The average estimates (A.E) and the associated MSE for $\alpha$

\begin{tabular}{|c|c|c|c|c|c|c|c|}
\hline \multirow[t]{2}{*}{$(n, T)$} & \multirow[t]{2}{*}{$R$} & & \multirow[t]{2}{*}{ MLE } & \multicolumn{2}{|c|}{ Bayes(Lindley) } & \multicolumn{2}{|c|}{ Bayes(MCMC) } \\
\hline & & & & prior 1 & prior 2 & prior 1 & prior 2 \\
\hline \multirow{6}{*}{$(30,1.5)$} & \multirow[t]{2}{*}{20} & A.E & 2.1113 & 2.1789 & 2.1068 & 2.1249 & 2.0919 \\
\hline & & MSE & 0.1848 & 0.2078 & 0.1646 & 0.1325 & 0.0953 \\
\hline & \multirow[t]{2}{*}{25} & A.E & 2.1091 & 2.0665 & 2.0948 & 2.1119 & 2.0992 \\
\hline & & MSE & 0.1503 & 0.1382 & 0.1035 & 0.1463 & 0.1210 \\
\hline & \multirow[t]{2}{*}{30} & A.E & 2.1057 & 2.1356 & 2.0855 & 2.1062 & 2.0826 \\
\hline & & MSE & 0.1519 & 0.1638 & 0.1231 & 0.1211 & 0.1163 \\
\hline \multirow{6}{*}{$(30,2.5)$} & \multirow[t]{2}{*}{20} & A.E & 2.1394 & 2.1244 & 2.0993 & 2.1253 & 2.1256 \\
\hline & & MSE & 0.1696 & 0.2187 & 0.1453 & 0.1218 & 0.0671 \\
\hline & \multirow[t]{2}{*}{25} & A.E & 2.0999 & 2.1224 & 2.0851 & 2.0687 & 2.0639 \\
\hline & & MSE & 0.1317 & 0.1708 & 0.1287 & 0.1054 & 0.0261 \\
\hline & \multirow[t]{2}{*}{30} & A.E & 2.0996 & 2.0466 & 2.1142 & 1.9790 & 1.9985 \\
\hline & & MSE & 0.1306 & 0.3042 & 0.1625 & 0.1142 & 0.0184 \\
\hline \multirow{6}{*}{$(50,1.5)$} & \multirow[t]{2}{*}{35} & A.E & 2.0586 & 2.0540 & 2.0209 & 2.0495 & 2.0262 \\
\hline & & MSE & 0.0820 & 0.0740 & 0.0727 & 0.0667 & 0.0318 \\
\hline & \multirow[t]{2}{*}{40} & A.E & 2.0633 & 2.0738 & 2.0604 & 2.0424 & 1.9912 \\
\hline & & MSE & 0.0791 & 0.1017 & 0.0752 & 0.0520 & 0.0275 \\
\hline & \multirow[t]{2}{*}{50} & A.E & 2.0623 & 2.0822 & 2.0609 & 2.0136 & 2.0124 \\
\hline & & MSE & 0.0764 & 0.0847 & 0.0809 & 0.0709 & 0.0152 \\
\hline \multirow{6}{*}{$(50,2.5)$} & \multirow[t]{2}{*}{35} & A.E & 2.0793 & 2.0795 & 2.0430 & 2.0196 & 2.0149 \\
\hline & & MSE & 0.0747 & 0.0741 & 0.0649 & 0.0481 & 0.0032 \\
\hline & \multirow[t]{2}{*}{40} & A.E & 2.0682 & 2.0799 & 2.0847 & 2.0085 & 2.0068 \\
\hline & & MSE & 0.0737 & 0.0722 & 0.0839 & 0.0296 & 0.0017 \\
\hline & \multirow[t]{2}{*}{50} & A.E & 2.0287 & 2.0525 & 2.0376 & 2.0013 & 2.0008 \\
\hline & & MSE & 0.0691 & 0.0891 & 0.0547 & 0.0154 & 0.0006 \\
\hline
\end{tabular}

choices of $R, T$ values. For a particular set of hybrid censored data, the MLEs and Bayes estimators are obtained as described before. Both non-informative and informative priors are used for the shape and scale parameters. In case of non-informative prior we take $a=b=$ $c=d=0$. We call it as Prior 1 . Note that as the hyper-parameters go to zero, the prior density becomes inversely proportional to its argument and also becomes improper. This density is commonly used as an improper prior for parameters in the range of zero to infinity. It should also be mentioned that when $a=b=0, \pi(\alpha \mid a, b)$ is not log-concave, but the posterior density function $g_{2}(\alpha \mid$ data $)$ is still log-concave. For the informative prior, we chose $a=2, b=1, c=d=1$. We call it as Prior 2. For computing different point estimators we generated 1000 samples from the IW distribution with $\alpha=2$ and $\lambda=1$. The averages and mean squared errors (MSE) of estimators of $\alpha$ and $\lambda$ are presented in Tables 1 and 2, respectively.

We also compute the $95 \%$ asymptotic confidence intervals based on MLEs. For comparison purposes, we compute the $95 \%$ HPD credible intervals from the Gibbs samples. We report the average confidence/credible lengths in Table 3. In Table 3, the first and second row represent the result for $\alpha$ and $\lambda$, respectively.

Some of the points are quite clear from Tables 1 and 2. In Tables 1 and 2, it is observed that the approximate Bayes estimators of unknown parameters based on Lindley's approximation match quite well with the Bayes estimators using MCMC method.

In most of the cases, the Bayes estimates obtained by using Lindley's approximation of $\lambda$ based on prior 1 perform better than the MLEs of $\lambda$, but while for $\alpha$ it is the other way. But in case of prior 2, the Bayes estimates using Lindley's approximation of $(\alpha, \lambda)$ perform marginally better than the MLEs for all cases considered. It is also observed that in most of cases the performance in terms of average bias and the MSE of Bayes estimates obtained by using MCMC procedure under Prior 1 are close to that of the corresponding behaviour of the MLEs or the Bayes estimates obtained by Lindley's approximations. But while using informative prior (Prior 2), the performance of the Bayes estimates by using MCMC are much better than the other estimates. Therefore, if the prior information are available, we should use the Bayes estimates, otherwise MLEs may be used to avoid the computational cost. 
Table 2: The average estimates (A.E) and the associated MSE for $\lambda$

\begin{tabular}{|c|c|c|c|c|c|c|c|}
\hline \multirow[t]{2}{*}{$(n, T)$} & \multirow[t]{2}{*}{$R$} & & \multirow[t]{2}{*}{ MLE } & \multicolumn{2}{|c|}{ Bayes(Lindley) } & \multicolumn{2}{|c|}{ Bayes(MCMC) } \\
\hline & & & & prior 1 & prior 2 & prior 1 & prior 2 \\
\hline \multirow{6}{*}{$(30,1.5)$} & 20 & A.E & 1.0464 & 0.9793 & 1.0119 & 1.0570 & 1.0537 \\
\hline & & MSE & 0.0514 & 0.0456 & 0.0472 & 0.0528 & 0.0425 \\
\hline & 25 & A.E & 1.0298 & 1.0164 & 1.0170 & 0.9971 & 0.9993 \\
\hline & & MSE & 0.0487 & 0.0406 & 0.0335 & 0.0452 & 0.0418 \\
\hline & 30 & A.E & 1.0264 & 1.0122 & 1.0216 & 1.0371 & 1.0283 \\
\hline & & MSE & 0.0461 & 0.0526 & 0.0418 & 0.0487 & 0.0395 \\
\hline \multirow{6}{*}{$(30,2.5)$} & 20 & A.E & 1.0107 & 1.0326 & 1.0071 & 1.0203 & 1.0237 \\
\hline & & MSE & 0.0467 & 0.0401 & 0.0445 & 0.0392 & 0.0359 \\
\hline & 25 & A.E & 1.0275 & 1.0055 & 1.0449 & 1.0159 & 0.9787 \\
\hline & & MSE & 0.0451 & 0.0591 & 0.0774 & 0.0309 & 0.0287 \\
\hline & 30 & A.E & 1.0282 & 1.0673 & 1.0342 & 0.9958 & 0.9864 \\
\hline & & MSE & 0.0437 & 0.0504 & 0.0418 & 0.0377 & 0.0261 \\
\hline \multirow{6}{*}{$(50,1.5)$} & 35 & A.E & 1.012 & 1.0002 & 1.0167 & 1.0423 & 1.0337 \\
\hline & & MSE & 0.0284 & 0.0258 & 0.0229 & 0.0276 & 0.0246 \\
\hline & 40 & A.E & 1.0153 & 0.9944 & 1.0144 & 1.0334 & 1.0221 \\
\hline & & MSE & 0.0271 & 0.0243 & 0.0226 & 0.0262 & 0.0241 \\
\hline & 50 & A.E & 1.0267 & 1.0131 & 1.0175 & 1.017 & 1.0263 \\
\hline & & MSE & 0.0266 & 0.0230 & 0.0278 & 0.0243 & 0.0240 \\
\hline \multirow{6}{*}{$(50,2.5)$} & 35 & A.E & 1.0159 & 1.0032 & 1.0013 & 1.0446 & 1.0373 \\
\hline & & MSE & 0.0260 & 0.0287 & 0.0259 & 0.0216 & 0.0207 \\
\hline & 40 & A.E & 1.0221 & 1.0037 & 0.9903 & 1.0245 & 1.0327 \\
\hline & & MSE & 0.0280 & 0.0255 & 0.0482 & 0.0219 & 0.0135 \\
\hline & 50 & A.E & 1.0228 & 1.0322 & 1.0253 & 1.0087 & 1.0074 \\
\hline & & MSE & 0.0262 & 0.0416 & 0.0261 & 0.0204 & 0.0018 \\
\hline
\end{tabular}

For all the methods, and for both the estimators, it is observed that for fixed $n$ as $R$ or $T$ increases in most of cases the average biases, and the MSE decrease, it verifies the consistency properties of the estimates.

Now, considering the confidence intervals and credible lengths, it is observed that the asymptotic results of the MLE work quite well. It can be seen that the average confidence lengths is quite close to the average credible intervals, mainly for large $n$ and $R$. But, in most of the cases, the average lengths of the credible intervals are slightly shorter than the confidence intervals. From Table 3 it is observed that the results obtained using informative priors are not significantly different than the corresponding results obtained using non-proper priors. Finally, note that Bayes estimates are most computationally expensive, followed by MLE.

\section{Illustration}

Consider the following data giving the maximum flood levels (in millions of cubic feet per second) of the Susquehenna River at Harrisburg, Pennsylvenia over 20 four-year periods (1890 - 1969). These data are taken from Dumonceaux and Antle (1973).
0.654
0.613
0.315
0.449
0.297
0.402
0.379
0.423
0.379
0.324
0.269
0.740
0.418
0.412
0.494
0.416
0.338
0.392
0.484
0.265

Before progressing, first we want to check whether the IW distribution fits the data or not. For this purpose, we have used the complete data. The MLEs and Bayes estimates of $(\alpha, \theta)$ based on the complete sample are $(4.3143,2.7905)$ and $(4.1861,2.7657)$, respectively. The Kolmogorov-Smirnov distance between the empirical distribution function and the fitted distribution functions when the parameters are obtained by MLEs, and the associated $p$-value are 0.1060 and 0.8557 , respectively. Since the $p$-value is quite high, we cannot reject the null hypothesis that the data are coming from the IW distribution. We have just plotted the empirical cumulative distribution function and the fitted cumulative distribution function in Figure 1. It shows that IWD fit the data very well. Now, we have created two artificially 
Table 3: The average confidence/credible lengths for the MLE and Bayes estimates of $\alpha$ and $\lambda$

\begin{tabular}{|c|c|c|c|c|}
\hline \multirow[t]{2}{*}{$(n, T)$} & \multirow[t]{2}{*}{$R$} & \multirow[t]{2}{*}{ MLE } & \multicolumn{2}{|c|}{ Bayes(MCMC) } \\
\hline & & & prior 1 & prior 2 \\
\hline \multirow{6}{*}{$(30,1.5)$} & \multirow[t]{2}{*}{20} & 1.4718 & 1.3986 & 1.3864 \\
\hline & & 0.8106 & 0.8230 & 0.79180 \\
\hline & \multirow[t]{2}{*}{25} & 1.4338 & 1.3891 & 1.3828 \\
\hline & & 0.7978 & 0.7663 & 0.7565 \\
\hline & \multirow[t]{2}{*}{30} & 1.4422 & 1.3911 & 1.3872 \\
\hline & & 0.7996 & 0.7508 & 0.7500 \\
\hline \multirow{6}{*}{$(30,2.5)$} & \multirow[t]{2}{*}{20} & 1.4384 & 1.4024 & 1.3835 \\
\hline & & 0.7891 & 0.8013 & 0.7812 \\
\hline & \multirow[t]{2}{*}{25} & 1.2730 & 1.1916 & 1.2014 \\
\hline & & 0.7873 & 0.7818 & 0.7416 \\
\hline & \multirow[t]{2}{*}{30} & 1.2533 & 1.1885 & 1.1762 \\
\hline & & 0.7788 & 0.7417 & 0.7469 \\
\hline \multirow{6}{*}{$(50,1.5)$} & \multirow[t]{2}{*}{35} & 1.1985 & 1.1842 & 1.1873 \\
\hline & & 0.6144 & 0.6327 & 0.5990 \\
\hline & \multirow[t]{2}{*}{40} & 1.0917 & 1.0916 & 1.0913 \\
\hline & & 0.6147 & 0.5986 & 0.5295 \\
\hline & \multirow[t]{2}{*}{50} & 1.0889 & 1.0862 & 1.0885 \\
\hline & & 0.6164 & 0.6069 & 0.6014 \\
\hline \multirow{6}{*}{$(50,2.5)$} & \multirow[t]{2}{*}{35} & 1.0450 & 1.0381 & 1.0399 \\
\hline & & 0.6022 & 0.5499 & 0.5427 \\
\hline & \multirow[t]{2}{*}{40} & 0.9934 & 1.0057 & 0.9831 \\
\hline & & 0.5968 & 0.5259 & 0.5082 \\
\hline & \multirow[t]{2}{*}{50} & 0.9483 & 0.9129 & 0.9210 \\
\hline & & 0.5958 & 0.5024 & 0.5169 \\
\hline
\end{tabular}

the type-I hybrid censored data sets from the above uncensored data set, using the following censoring schemes:

Scheme 1: $R=18, T=0.5$.

In this scheme, it is observed that the $R$-th failure does not take place before time point $T$. For this scheme, the hybrid censored sample is:

$$
\begin{array}{llllllllll}
0.265 & 0.269 & 0.297 & 0.315 & 0.324 & 0.338 & 0.379 & 0.379 & 0.392 & 0.402 \\
0.412 & 0.416 & 0.418 & 0.423 & 0.449 & 0.484 & 0.494 & & &
\end{array}
$$

From the above sample data, the MLEs of $\alpha$ and $\theta$ are 4.2726 and 2.6565, respectively. Since we do not have any prior information available, we use non-informative priors, i.e., $a=b=c=d=0$ on both $\alpha$ and $\theta$ to compute Bayes estimatores. Now using Algorithm of section 4.2, we generate $1000 \mathrm{MCMC}$ samples and based on them we compute the Bayes estimates of $\alpha$ and $\theta$ as 4.5665 and 2.8148, respectively. The $95 \%$ asymptotic confidence intervals of $\alpha$ and $\theta$ based on the empirical Fisher information matrix are $(2.7207,5.8244)$ and $(2.3623,2.9507)$ respectively. Moreover, the 95\% HPD credible intervals of $\alpha$ and $\theta$ are $(2.4603,5.2454)$, and $(2.2977,2.8928)$, respectively.

Scheme 2: $R=14, T=0.45$. In this scheme, it is observed that the $R$-th failure took place before $T$. In this case, the hybrid sample is:

$$
\begin{array}{llllllllll}
0.265 & 0.269 & 0.297 & 0.315 & 0.324 & 0.338 & 0.379 & 0.379 & 0.392 & 0.402 \\
0.412 & 0.416 & 0.418 & 0.423 & & & & & &
\end{array}
$$

Based on the sample, the MLEs and Bayes estimates of $\alpha$ and $\theta$ are $(3.6933,2.5446)$ and $(3.8607,2.7158)$ respectively. The $95 \%$ asymptotic confidence intervals of $\alpha$ and $\theta$ are $(2.2233$, $5.1635)$ and $(2.2088,2.8804)$ respectively. We also compute the $95 \%$ HPD credible intervals of $\alpha$ and $\theta$ and they are $(2.3481,5.2153)$, and $(2.2718,3.0145)$, respectively. 


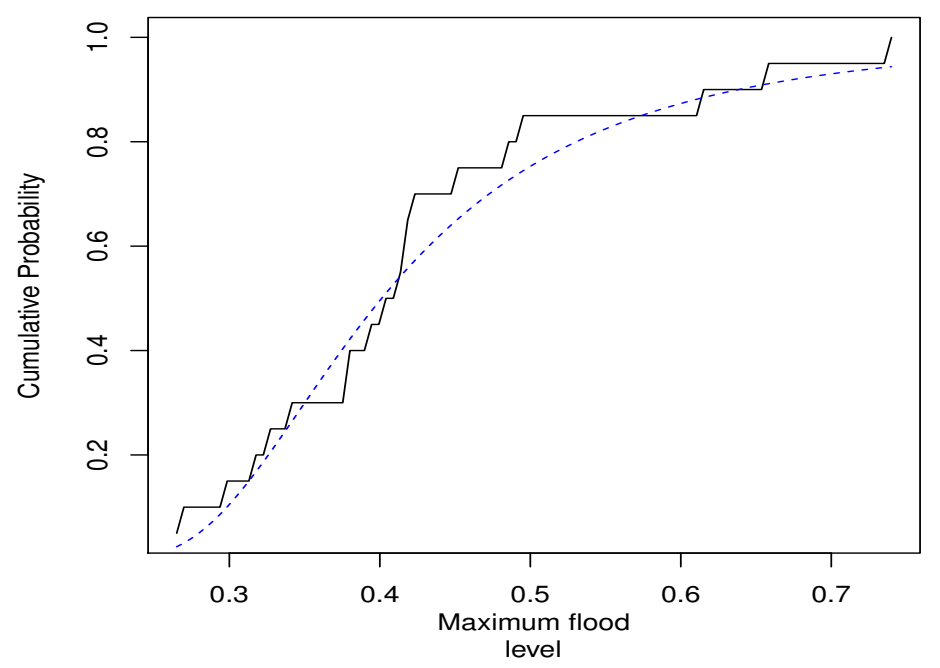

Figure 1: The empirical and fitted distributions

\section{Conclusion and discussion}

The inverse Weibull distribution is found to be very appropriate over Weibull distribution when data indicates the non-monotone hazard rate. There are various real life examples where data don't shows the monotone hazard rate. For example, Langlands, Pocock, Kerr, and Gore (1997) have studied breast cancer data and observed that the mortality increases initially, reaches to a peak after some time and then declines slowly. Such types of data can be modelled through inverse Weibull distribution. Since last decade, many authors have studied the classical as well as Bayesian estimation procedures for the estimation of the unknown parameters of inverse Weibull distribution under different types censoring schemes. We mentioned some references in the Introduction for more information. By the way, estimation of the inverse Weibull distribution parameters under type-I hybrid censoring has not been developed yet.

In this paper we considered the classical and Bayesian inference of the inverse Weibull distribution based on type-I hybrid censored data. The maximum likelihood estimators of the parameters can be obtained by using an iterative procedure. Hence the Bayesian inference seems to be the natural choice for the analysis of certain survival data. The prior belief of the model was represented by the independent gamma priors on the shape and scale parameters. The squared error loss function was used as it is appropriate when large errors of the estimation are considered to be more serious compared to other loss functions. It was observed that the Bayes estimators and the HPD credible intervals can not be obtained in explicit form. We proposed two approximations which can be implemented very easily. We compared the performance of the Bayes estimators with the MLEs by Monte Carlo simulations and a real life example. It was observed that the performances are quite satisfactory.

In practice, it is very important to choose the optimum censoring scheme from a class of possible schemes to obtain the highest amount of information about the unknown parameters. Here possible schemes mean, for fixed sample size $n$, different choices of $R$ and $T$. Indeed, introducing an effective procedure to obtain the optimum censoring scheme can be an interesting topic for future research. Also, it may be of interest to obtain the one and two sample predictive posteriors of future observations in the presence of type-I hybrid censored sample. In addition, the inferential results of some life time models under type-I hybrid censored data with random removals remain open. More work is needed in those directions. 


\section{Acknowledgments}

The authors would like to thank the Editor and anonymous reviewer for their helpful remarks and corrections which improved the original manuscript.

\section{References}

Ateya SF (2017). "Estimation under Inverse Weibull Distribution Based on Balakrishnan's Unified Hybrid Censored Scheme." Communications in Statistics-Simulation and Computation, 46(5), 3645-3666.

Ateya SF (2020). "Statistical Inferences under Inverse Weibull Distribution Based on Generalized Type-II Progressive Hybrid Censoring Scheme." Journal of Statistical Applications and Probability Letters, 7(2), 67-75.

Balakrishnan N, Shafay AR (2012). "One- and Two-sample Bayesian Prediction Intervals Based on Type-II Hybrid Censored Data." Annals of Statistics, 41, 1511-1531.

Chen MH, Shao QM (1999). "Monte Carlo Estimation of Bayesian Credible and HPD Intervals." Journal of Computational and Graphical Statistics, 8(1), 69-92.

Devroye L (1984). "A Simple Algorithm for Generating Random Variates with a Log-concave Density." Computing, 33(3-4), 247-257.

Dumonceaux R, Antle CE (1973). "Discrimination between the Log-normal and the Weibull Distributions." Technometrics, 15(4), 923-926.

Epstein B (1954). "Truncated Life Tests in the Exponential Case." Annals of Statistics, 25, $555-564$.

Gupta RD, Kundu D (1988). "Hybrid Censoring Schemes with Exponential Failure Distribution." Annals of Statistics, 27, 3065-3083.

Hyun S, Lee J, Yearout R (2016). "Parameter Estimation of Type-I and Type-II Hybrid Censored Data from the Log-Logistic Distribution." Industrial and Systems Engineering Review, 4(1), 37-44.

Kundu D (2007). "On Hybrid Censored Weibull Distribution." Annals of Statistics, 137, $2127-2142$.

Kundu D, Howlader H (2010). "Bayesian Inference and Prediction of the Inverse Weibull Distribution for Type-II Censored Data." Computational Statistics $\&$ Data Analysis, 54(6), $1547-1558$.

Kundu D, Pradhan B (2009). "Estimating the Parameters of the Generalized Exponential Distribution in Presence of Hybrid cCnsoring." Communications in Statistics - Theory and Methods, 38(12), 2030-2041.

Langlands AO, Pocock SJ, Kerr GR, Gore SM (1997). "Long-term Survival of Patients with Breast Cancer: A Study of the Curability of the Disease." British Medical Journal, 2(6200), $1247-1251$.

Lindley DV (1980). "Approximate Bayesian Methods." Trabajos de estadística y de investigación operativa, 31(1), 223-245.

Raqab MZ, Madi MT (2005). "Bayesian Inference for the Generalized Exponential Distribution." Journal of Statistical Computation and Simulation, 75(10), 841-852. 
Rastogi MK, Tripathi YM (2013). "Inference on Unknown Parameters of a Burr Distribution under Hybrid Censoring." Statistical Papers, 54(3), 619-643.

Singh S, Tripathi YM (2015). "Bayesian Estimation and Prediction for a Hybrid Censored Lognormal Distribution." IEEE Transactions on Reliability, 65(2), 782-795.

Singh SK, Singh U, Sharma VK (2013). "Bayesian Analysis for Type-II Hybrid Censored Sample from Inverse Weibull Distribution." International Journal of System Assurance Engineering and Management, 4(3), 241-248.

Tripathi YM, Rastogi MK (2016). "Estimation Using Hybrid Censored Data from a Generalized Inverted Exponential Distribution." Communications in Statistics-Theory and Methods, 45(16), 4858-4873.

\section{Appendix A}

For the two-parameter case, using the notation $\left(\lambda_{1}, \lambda_{2}\right)=(\alpha, \lambda)$, the Lindley's approximation can be written as,

$$
\hat{g}=g\left(\hat{\lambda}_{1}, \hat{\lambda}_{2}\right)+\frac{1}{2}\left[A+l_{30} B_{12}+l_{03} B_{21}+l_{21} C_{12}+l_{12} C_{21}\right]+p_{1} A_{12}+p_{2} A_{21},
$$

where,

$$
\begin{gathered}
A=\sum_{i=1}^{2} \sum_{j=1}^{2} w_{i j} \tau_{i j}, \quad l_{i j}=\frac{\partial^{i+j} L\left(\lambda_{1}, \lambda_{2}\right)}{\partial \lambda_{1}^{i} \partial \lambda_{2}^{j}}, i, j=0,1,2,3, i+j=3, \quad p_{i}=\frac{\partial p}{\partial \lambda_{i}}, \\
w_{i}=\frac{\partial g}{\partial \lambda_{i}}, \quad w_{i j}=\frac{\partial^{2} g}{\partial \lambda_{i} \partial \lambda_{j}}, \quad p=\ln \pi\left(\lambda_{1}, \lambda_{2}\right), \quad A_{i j}=w_{i} \tau_{i i}+w_{j} \tau_{j i} \\
B_{i j}=\left(w_{i} \tau_{i i}+w_{j} \tau_{i j}\right) \tau_{i i}, C_{i j}=3 w_{i} \tau_{i i} \tau_{i j}+w_{j}\left(\tau_{i i} \tau_{j j}+2 \tau_{i j}^{2}\right),
\end{gathered}
$$

here $\mathrm{L}(.,$.$) is the log-likelihood function of the observed data, \pi\left(\lambda_{1}, \lambda_{2}\right)$ is the joint prior density function of $\left(\lambda_{1}, \lambda_{2}\right)$ and $\tau_{i j}$ is the $(\mathrm{i}, \mathrm{j})$-th element of the inverse of the observed Fisher information matrix. Moreover, $\hat{\lambda}_{1}$ and $\hat{\lambda}_{2}$ are the MLEs of $\left(\lambda_{1}, \lambda_{2}\right)$, respectively and all the quantities are evaluated at $\left(\hat{\lambda}_{1}, \hat{\lambda}_{2}\right)$.

Now we have

$$
L(\alpha, \lambda \mid x)=\alpha^{r} \lambda^{r} e^{-\lambda \sum_{i=1}^{r} x_{i}^{\alpha}} \prod_{i=1}^{r} x_{i}^{\alpha+1}\left(1-e^{-\lambda u^{-\alpha}}\right)^{n-r} .
$$


Therefore, we obtain

$$
\begin{aligned}
& l_{03}=\frac{2 r}{\hat{\lambda}^{3}}+\frac{(n-r) u^{-3 \hat{\alpha}} e^{-\hat{\lambda} u^{-\hat{\alpha}}}}{1-e^{-\hat{\lambda} u^{-\hat{\alpha}}}}+\frac{3(n-r) u^{-3 \hat{\alpha}} e^{-2 \hat{\lambda} u^{-\hat{\alpha}}}}{\left(1-e^{-\hat{\lambda} u^{-\hat{\alpha}}}\right)^{2}}+\frac{2(n-r) u^{-3 \hat{\alpha}} e^{-3 \hat{\lambda} u^{-\hat{\alpha}}}}{\left(1-e^{-\hat{\lambda} u^{-\hat{\alpha}}}\right)^{3}}, \\
& l_{30}=\frac{2 r}{\hat{\alpha}^{3}}-\frac{(n-r) \hat{\lambda} u^{-\hat{\alpha}}(\ln u)^{3} e^{-\hat{\lambda} u^{-\hat{\alpha}}}\left(1-3 \hat{\lambda} u^{-\alpha}+\hat{\lambda}^{2} u^{-2 \alpha}\right)}{1-e^{-\hat{\lambda} u^{-\alpha}}} \\
& +\frac{3(n-r) \hat{\lambda}^{2} u^{-2 \hat{\alpha}} \ln u^{3} e^{-2 \hat{\lambda} u^{-\hat{\alpha}}}\left(1-\hat{\lambda} u^{-\hat{\alpha}}\right)}{\left(1-e^{-\hat{\lambda} u^{-\hat{\alpha}}}\right)^{2}}-\hat{\lambda} \sum_{i=1}^{r} x_{i}^{\hat{\alpha}}\left(\ln x_{i}\right)^{3} \\
& -\frac{2(n-r) \hat{\lambda}^{3} u^{-3 \hat{\alpha}} \ln u^{3} e^{-3 \hat{\lambda} u^{-\hat{\alpha}}}\left(1-\hat{\lambda} u^{-\hat{\alpha}}\right)}{\left(1-e^{-\hat{\lambda} u^{-\hat{\alpha}}}\right)^{3}}, \\
& l_{12}=\frac{(n-r) u^{-2 \hat{\alpha}} \ln (u) e^{-\hat{\lambda} u^{-\hat{\alpha}}}\left(2-\hat{\lambda} u^{-\hat{\alpha}}\right)}{1-e^{-\hat{\lambda} u^{-\hat{\alpha}}}}-\frac{2(n-r) \lambda u^{-3 \hat{\alpha}} \ln (u) e^{-3 \hat{\lambda} u^{-\hat{\alpha}}}}{\left(1-e^{-\hat{\lambda} u^{-\hat{\alpha}}}\right)^{3}} \\
& +\frac{(n-r) u^{-2 \hat{\alpha}} \ln (u) e^{-2 \hat{\lambda} u^{-\hat{\alpha}}}\left(2-3 \hat{\lambda} u^{-\hat{\alpha}}\right)}{\left(1-e^{-\hat{\lambda} u^{-\hat{\alpha}}}\right)^{2}}, \\
& l_{21}=-\sum_{i=1}^{r} x_{i}^{\hat{\alpha}}\left(\ln x_{i}\right)^{2}+\frac{(n-r) u^{-\hat{\alpha}}(\ln u)^{2} e^{-\hat{\lambda} u^{-\hat{\alpha}}}\left(1-3 \hat{\lambda} u^{-\hat{\alpha}}+\hat{\lambda}^{2} u^{-2 \hat{\alpha}}\right)}{1-e^{-\hat{\lambda} u^{-\hat{\alpha}}}} \\
& -\frac{3(n-r) \hat{\lambda} u^{-2 \hat{\alpha}} \ln (u)^{2} e^{-2 \hat{\lambda} u^{-\hat{\alpha}}}\left(1-\hat{\lambda} u^{-\hat{\alpha}}\right)}{\left(1-e^{-\hat{\lambda} u^{-\hat{\alpha}}}\right)^{2}} \\
& +\frac{2(n-r) \hat{\lambda}^{2} u^{-3 \hat{\alpha}} \ln u^{2} e^{-3 \hat{\lambda} u^{-\alpha}}}{\left(1-e^{-\hat{\lambda} u^{-\hat{\alpha}}}\right)^{3}} .
\end{aligned}
$$

The elements of the Fisher information matrix are obtained in section 4. By using (6), $\tau_{i j}=V^{i j}$, where $i, j=1,2$.

Now when $g(\alpha, \lambda)=\alpha$, then

$$
w_{1}=1, w_{2}=0, \quad w_{i} j=0, \quad \text { for } i, j=1,2 .
$$

Therefore,

$$
\begin{gathered}
A=0, B_{12}=\tau_{11}^{2}, B_{21}=\tau_{21} \tau_{22}, C_{12}=3 \tau_{11} \tau_{12}, \\
C_{21}=\left(\tau_{22} \tau_{11}+2 \tau_{21}^{2}\right), A_{12}=\tau_{11}, A_{21}=\tau_{12} .
\end{gathered}
$$

Now the first part of Lindley's approximation follows by using

$$
p_{1}=\frac{d-1}{\alpha}-c, p_{1}=\frac{b-1}{\lambda}-a .
$$

For the second part, note that $g(\alpha, \lambda)=\lambda$, then

$$
w_{1}=0, w_{2}=1, w_{i} j=0, \text { for } i, j=1,2 .
$$

Therefore,

$$
\begin{gathered}
A=0, B_{12}=\tau_{12} \tau_{11}, B_{21}=\tau_{22}^{2}, C_{12}=\left(\tau_{11} \tau_{22}+2 \tau_{12}^{2}\right) \\
C_{21}=3 \tau_{22} \tau_{21}, A_{12}=\tau_{21}, A_{21}=\tau_{22} .
\end{gathered}
$$

therefore the second part follows immediately. 


\section{Appendix B}

The conditional density of $\alpha$ given the data is

$$
g_{2}(\alpha \mid \text { data }) \propto \frac{1}{\left(d+\sum_{i=1}^{r} x_{i}^{\alpha}\right)^{r+c}} \alpha^{a+r-1} e^{-b \alpha} \prod_{i=1}^{r} x_{i}^{\alpha+1} .
$$

The logarithm of $g_{2}(\alpha \mid$ data $)$ without the additive constant is

$$
\ln g_{2}(\alpha \mid d a t a)=-(r+c) \ln \left(d+\sum_{i=1}^{r} x_{i}^{\alpha}\right)+(a+r-1) \ln (\alpha)-b \alpha+(\alpha+1) \sum_{i=1}^{r} \ln \left(x_{i}\right),
$$

Using Lemma 1 of Kundu (2007), it follows that

$$
\frac{d}{d \alpha^{2}} \ln \left(\sum_{i=1}^{r} x_{i}^{\alpha}+d\right) \geq 0
$$

Therefore the result follows.

\section{Affiliation:}

Mohammad Kazemi

School of Mathematical Scinces

University of Guilan

Rasht, Guilan, Iran

E-mail: m.kazemie64@yahoo.com

\section{Austrian Journal of Statistics}

published by the Austrian Society of Statistics

Volume 50

July 2021 http://www.ajs.or.at/

http://www.osg.or.at/

Submitted: 2020-04-04

Accepted: 2020-08-05 